\title{
3.5 Barrieren, Hemmschwellen und Gatekeeper
}

\begin{abstract}
Barrieren - im Sinne fehlender Angebote und Infrastrukturen - oder Hemmschwellen - im Sinne erlernter, tradierter Verhaltensweisen - verhindern bislang häufig die transparente Beschreibung, Bereitstellung und Nachnutzung von Forschungsdaten. Um dies $\mathrm{zu}$ ändern, sind alle Forschenden durch individuelles Handeln, aber auch die Gatekeeper des Wissenschaftsbetriebes gefordert. Letztere können durch den Aufbau von Infrastrukturen zur Unterstützung der Forschenden, durch finanzielle und personelle Anreize und Erleichterungen bei der praktischen Umsetzung des Forschungsdatenmanagements, aber auch durch die strukturell abgesicherte Belohnung der Aufbereitung und freien Zugänglichkeit von Forschungsdaten in Form von wissenschaftlicher Reputation dazu beitragen, dass Barrieren entfallen und Verhaltensänderungen die Hemmschwellen marginalisieren.
\end{abstract}

\section{Einleitung}

Eigentlich wäre alles so einfach: Wissenschaftlerinnen und Wissenschaftler forschen, dabei nutzen, sammeln oder produzieren sie Forschungsdaten (FD), versehen diese mit entsprechenden Metadaten und stellen sie über ihnen geeignet erscheinende Repositorien zur Nachnutzung unter definierten rechtlichen Bedingungen zur Verfügung. Die Realität - in Deutschland wie auch international - ist jedoch anders: Bislang machen nur wenige Wissenschaftlerinnen und Wissenschaftler ihre Daten frei zugänglich. Die Frage ist: Warum?

Empirische Untersuchungen und Erfahrungsberichte aus den letzten Jahren haben hierfür vielfältige Ursachen deutlich werden lassen: Noch fehlt häufig die subjektive Bereitschaft und Kompetenz bei den einzelnen Forschenden für die aus wissenschaftlicher wie forschungspolitischer Sicht wünschenswerte systematische Erfassung, das Kuratieren und die Bereitstellung von FD zur transparenten Absicherung guter wissenschaftlicher Praxis. Für die Nachnutzung von FD ist aber beides unabdingbar.

Neben personenbezogenen Gründen zeigt aber auch das Wissenschaftssystem mit seinen Interessengruppen und Strukturen noch erhebliche Defizite, den Wandel zum gewünschten Forschungsdatenmanagement (FDM) zu befördern: Die grundsätzlichen Vorgaben und formellen Regeln, aber auch die passende Infrastruktur, zugeordnete Workflows, kompetenzfördernde Anleitungen oder Beratungen und die notwendige rechtlichen Absicherung für eine transparente Bereitstellung und Nach- 
nutzung von FD werden erst allmählich den forschungspolitischen Zielsetzungen

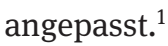

Vorreiter bei der praktischen Umsetzung dieser Zielsetzung sind Forschungskontexte, in denen kooperativ und ggf. auch kollaborativ gearbeitet wird. Häufig arbeiten hier Forschende aus universitären und außeruniversitären Einrichtungen zusammen, die auf systematisch ausgebaute Forschungsinfrastrukturen zurückgreifen wollen und z. T. auch schon können.

In Fachdisziplinen, die schon lange in solchen Strukturen arbeiten - z. B. Klimaforschung, Astrophysik oder Teilchenphysik -, sind daher die Hemmschwellen und Barrieren zur Bereitstellung und Nachnutzung von FD relativ gering. ${ }^{2}$ In anderen - wie z. B. den Digital Humanities $(\mathrm{DH})$ - wirken sie jedoch noch stärker, vermutlich aber dort am wenigsten, wo wiederum kollaborativ z. B. in virtuellen Forschungsumgebungen gearbeitet wird. Indiz hierfür ist der Umstand, dass gerade aus den DH-Forschungsverbünden heraus Lösungen für eine FDM-adäquate Infrastruktur entwickelt werden. ${ }^{3}$

Trotz solcher Vorreiter ist FDM für viele Forschende ein von ihnen eingefordertes Desiderat, dem tradierte Verhaltensweisen, aber auch fehlende Infrastrukturen entgegenstehen. Aus dieser Einschätzung leitet sich die Binnenstruktur des vorliegenden Beitrags ab: Er skizziert zuerst aus der Perspektive der einzelnen Forschenden Hinderungsgründe, ihre Forschungsprozesse und die dabei erstellten bzw. ermittelten FD mittels FDM transparent zu machen. Ob diese Hinderungsgründe als Hemmschwellen (im Sinne erlernter, tradierter Verhaltensweisen) oder als Barrieren (im Sinne fehlender Angebote und Infrastrukturen) wahrgenommen werden, dürfte subjektiv z. T. variieren. $\mathrm{Zu}$ vermuten ist, dass Hemmschwellen und Barrieren von den meisten als nicht weiter differenziertes Konglomerat zur Verhinderung eines offenen Umgangs mit FD wahrgenommen werden.

In einem zweiten Schritt werden dann mögliche Maßnahmen angesprochen, mit denen - gegliedert nach den jeweiligen Gruppen von Gatekeepern - die Wissenschaftsgemeinschaft(en) mit ihren Institutionen sowie Strukturen und hierbei insbesondere mit ihren Regularien und ihren Anerkennungssystemen die konkrete Bereitschaft zur Bereitstellung und Nachnutzung von FD befördern können.

1 Vgl. z. B. Sane und Edelstein 2015, 6-16, sowie diverse Beiträge in diesem Buch.

2 Vgl. Neuroth et al. 2012, 295-310; Zuiderwijk und Spiers 2019, 231-232.

3 Vgl. z. B. Cremer, Klaffki und Steyer 2019, 120 f. 


\section{Forschende und ihre Gründe, Daten nicht transpa- rent zu machen}

Im Mittelpunkt des Forschungsprozesses stehen die Forschenden als Individuen mit ihrer Motivation Lösungen für Forschungsfragen zu finden und mit diesen Lösungen wissenschaftliches Renommee zu gewinnen. Traditionell stand und steht im Forschungsprozess das Individuum und seine durch Kreativität und / oder geduldige Analyse auf der Grundlage transparenter methodischer Überlegungen erzielte Leistung im Mittelpunkt. Entsprechend war und ist das Wissenschaftssystem in den meisten Bereichen auf Einzelpersonen und die von ihnen erzielten und ggf. erstmalig veröffentlichten Ergebnisse ausgerichtet. Dies gilt in Form der Fokussierung auf inspirierende, leitende und organisatorisch verantwortliche Wissenschaftlerinnen und Wissenschaftler selbst dort, wo Gruppen von Forschenden unter deren Gesamtverantwortung kooperativ und kollaborativ arbeiten.

Entsprechend werden normalerweise für die erzielten Ergebnisse wissenschaftsbezogene Belohnungen wie Titel, Ehrungen, Positionen und insbesondere Forschungsgelder zugeteilt. Mit diesen können die so unterstützten Forschenden dann im Idealfall erfolgreich weitere herausragende Leistungen und Ergebnisse erzielen. In einem solchen, stark auf persönliche Leistung und Karriere ausgerichteten System sind FD, die u. U. mühsam und in langwierigen Prozessen erhoben wurden, ein Kapital, das nur dann transparent gemacht wird, wenn es schon umfassend ausgewertet und zu Publikationen verarbeitet wurde oder andere Notwendigkeiten bestehen, es bereitzustellen. Dies gilt insbesondere für angehende Wissenschaftlerinnen und Wissenschaftler, die sich im Rahmen von Qualifizierungsarbeiten (z. B. Master, Promotion oder Habilitation) sowie Projekten profilieren wollen. Die traditionelle Hemmschwelle zu überwinden und FD nicht für sich zu behalten, bedarf daher intensiver Motivation. Diese wird ganz wesentlich davon beeinflusst, inwieweit die Bereitstellung der Daten sich in Formen der Anerkennung und Belohnung in der Wissenschaftswelt niederschlägt. ${ }^{4}$

Die subjektiven, durchaus auch psychologischen Aspekte der bislang erkennbaren Zurückhaltung insbesondere von Forschenden, die nicht im Verbund und kollaborativ arbeiten, erlangen in der Forschung zum FDM deutliche Beachtung. ${ }^{5}$ Gleichzeitig sind sie aber auch Gegenstand von internationalen Projektaktivitäten, die z. B. im Teilprojekt GO CHANGE der Initiative GO FAIR ${ }^{6}$ angegangen werden.

4 Vgl. Lucraft et al. 2019, 12-14.

5 Vgl. z. B. Linek et al. 2017, 1-24.

6 S. https://www.go-fair.org/go-fair-initiative/go-change/ und den Beitrag von Linne et al., Kap. 3.2 in diesem Praxishandbuch. Letztes Abrufdatum der Internet-Dokumente ist der 15.11.2020. 
Mangelnde Kompetenz und Erfahrung im Umgang mit FD sind immer wieder ein zentrales Hindernis, Forschungsprozesse und die dabei gewonnenen bzw. genutzten FD im Sinne eines transparenten FDM adäquat zu erfassen und aufzubereiten. Die entsprechenden Defizite führen dazu, dass die einer Veröffentlichung zugrundeliegenden FD häufig nicht publiziert, also auch nicht intersubjektiv überprüft oder ggf. für nachfolgende Forschungen genutzt werden können. Unklarheiten bzgl. dessen, was überhaupt im konkreten Kontext FD sind, Unklarheiten bei der Erhebung, der Dokumentation des Vorgehens und der Beschreibung gewonnener Daten mittels Metadaten sowie rechtliche Unsicherheiten können auch die Bereitwilligsten daran hindern, die Veröffentlichung ihrer FD zu ermöglichen. Jüngste Forschungen zeigen, dass 65 Prozent aller Forschenden entsprechenden Beratungsbedarf für sich reklamieren. Sie sehen ihre Mitforschenden z. B. in Fachgesellschaften, aber auch Verlage und Bibliotheken in der Pflicht, entsprechende Dienste bereitzustellen. ${ }^{7}$ Diese subjektive Unsicherheit und die damit verbundenen objektiven Defizite konkretisieren sich $\mathrm{u}$.a. in den nachfolgend erläuterten, ausgewählten Gründen, seine FD nicht bereit zu stellen. ${ }^{8}$

\subsection{Verlust der Datenhoheit}

Die Datenhoheit über die selbst erhobenen FD zu verlieren, ist wohl die größte Befürchtung und damit eine klassische Hemmschwelle von Forschenden: Ihre Daten sind aus ihrer Sicht noch nicht hinreichend ausgewertet und in einer nicht aufbereiteten, d.h. u.a. ohne erläuternde Metadaten versehenen Form für Dritte nicht verständlich. Dies mag z.T. bewusst provoziert werden, denn schließlich sind Dritte u. U. Konkurrenten im Wissenschaftsbetrieb und es soll verhindert werden, dass diese sich die eigene Vorarbeit zunutze und damit womöglich Karriere machen.

Eine zumeist unausgesprochene, aber vermutlich gerade bei Qualifizierungsarbeiten durchaus berechtigte subjektive Befürchtung kann zudem sein, dass „Schwächen der Datenerhebung und -analyse sichtbar werden“9 oder dass erkennbar wird, dass Daten sogar manipuliert sind. ${ }^{10}$

Warum auch immer eine Aufbereitung der Daten unterbleibt: Die Furcht vor dem Verlust der Datenhoheit ist bislang ein im Wissenschaftssystem durchaus ak-

7 Vgl. Lucraft et al. 2019, 18.

8 Eine viele Aspekte abdeckende Übersicht zu möglichen Gründen, FD nicht zu publizieren, hat Kaden 2018 auf der Grundlage einer Diskussion beim Open-Science-Bar-Camp des Leibniz Forschungsverbunds Science 2.0 am 12.03.2018 zusammengestellt.

9 Kaden 2018.

10 Vgl. zu dieser These die Untersuchung von Wicherts, Bakker und Molenar 2011, den Beitrag von Wiarda 2019 sowie insgesamt zu diesem Themenkomplex die fortwährend aktualisierte Sammlung zurückgezogener Paper unter https://retractionwatch.com/. 
zeptiertes Argument. Im Wesentlichen ist es dem großen Publikationsdruck gerade der sich in traditioneller Form profilierender Forschenden geschuldet.

\subsection{Fehlender und unzureichend umgesetzter Datenmanagement- plan}

Um Daten und ihre Erhebung für Dritte nachvollziehbar zu machen, sollten schon bei der Planung des Forschungsvorhabens das Konzept der Datenerhebung, die dabei zu erfassenden Metadaten, die Nutzungsanforderungen und -möglichkeiten sowie die damit verbundenen rechtlichen Aspekte in einem Gesamtkonzept, dem sog. Datenmanagementplan (DMP), festgehalten werden. Der Aufwand, diesen umzusetzen, ist umso geringer, je früher und systematischer die Planung und Umsetzung erfolgt. So kann ggf. das Erfassen von forschungsdatenbezogenen Metadaten (teil-) automatisiert erfolgen.

Die Erstellung und Umsetzung eines DMP wird wegen entsprechender Anforderungen der Forschungsförderer ${ }^{11}$ selbstverständlicher. Dennoch werden insbesondere Forschungsvorhaben Einzelner häufig mangels Kenntnis des Konzeptes oder wegen des vermeintlich zu hohen Aufwands noch ohne DMP realisiert. Dieses Defizit wird erst dann offenbar, wenn weiterführende Forschungsaktivitäten unter Nachnutzung der zuvor gewonnenen Daten anstehen. Dann aber fehlen die die Datenerhebung dokumentierenden Metadaten ebenso wie die rechtliche Absicherung zu deren Nachnutzung (s. u.) in einem weiteren Forschungskontext.

\subsection{Aufwand}

Abhängig vom Forschungsgebiet und den eingesetzten Methoden, aber auch abhängig von der Regeltreue, mit der die im Datenmanagementplan als notwendig deklarierten Maßnahmen zur Qualitätssicherung, Beschreibung und dem Kuratieren von FD umgesetzt werden, entsteht $u$. U. ein erheblicher zeitlicher und ggf. auch finanzieller Aufwand. Dieser wird nachvollziehbarerweise von vielen Forschenden bislang häufig noch nicht als integraler Bestandteil, sondern als zusätzlicher Aufwand im Rahmen ihrer Forschungsaktivitäten wahrgenommen, der sie vom „eigentlichen Forschen“ abhält.

Die dabei anfallenden Aufgaben sind durchaus umfangreich, wie eine entsprechende Auflistung vor dem Hintergrund praktischer Erfahrungen in der Max-WeberStiftung deutlich macht:

11 S. den Beitrag von Putnings, Kap. 1.3 in diesem Praxishandbuch. 
- Anamnese der Daten (durch ein Dateninterview)

- Appraisal des Datensatzes (Auswahl der Dateien und Informationspakete)

- Explikation (Variablenbezeichnung, Abkürzungen)

- Konversion in standardisierte, nicht-proprietäre Formate (xls in csv)

- Wahl des Dateiformats und Durchführung der Formatvalidierung (TIFF)

- Transformation und Strukturierung (Texte in TEI-P5)

- Automatisierte Anreicherung (Named Entity Recognition)

- Validierung gegen Standards (FAIR Data Prinzipien)

- Wahl des Datenrepositoriums (via re3data.org)

- Ingest (Account, Paketierung, Uploadprozess)

- $\quad$ Lizenzvergabe (kompatibel mit der Offen-Definition und ... FAIR-Prinzipien)

- Metadatenvergabe (DataCite-Metadata-Schema)

- Dokumentation (natursprachliche Sammlungsbeschreibung) ${ }^{12}$

Die Tragweite unzureichend dokumentierter und kuratierter FD für die Wahrnehmbarkeit der erzielten Forschungsergebnisse wird zumeist erst zu spät erkannt. Dokumentation im Nachhinein erweist sich aber als zusätzlich aufwendig. Der Verzicht auf eine Veröffentlichung der Daten erscheint dann vordergründig als der einfachere Weg. Dies auch deshalb, weil bislang im Wissenschaftsbetrieb noch häufig die textbasierte Publikation allein als hinreichender wissenschaftlicher Output angesehen und anerkannt wird.

Dabei ist es - abhängig von der Förderorganisation - durchaus möglich, Kosten für die Aufbereitung von Daten und für die Nutzung existierender Infrastrukturen zu beantragen. ${ }^{13}$ Da solche Anreize bislang nur bei manchen Forschungsförderern zugänglich waren, bedarf es hier umso mehr eines grundlegenden Bewusstseinswandels, um in allen Bereichen die Dokumentation und das Kuratieren von FD als selbstverständlichen und unabdingbaren Teil jeglicher Forschung zu verstehen.

12 Cremer, Klaffki und Steyer 2019, 121-122 - ohne die Fußnotennummern im Original. Auf das von dieser Autorengruppe vorgeschlagene Konzept der Unterstützung durch eine sog. Forschungsdatenredaktion wird später im Zusammenhang mit möglichen Maßnahmen von Verlagen eingegangen. 13 So beispielsweise bei der Deutschen Forschungsgemeinschaft (DFG): „Projektspezifische Kosten, die im Rahmen eines wissenschaftlichen Projekts bei der Aufbereitung von Forschungsdaten für eine Anschlussnutzung bzw. für die Überführung von Forschungsdaten in existierende Infrastrukturen entstehen, können mit dem Antrag bei der DFG eingeworben werden.“ Deutsche Forschungsgemeinschaft 2020. Ein grundsätzlich ähnliches Verständnis leitet die internationale Initiative der Forschungsförderer, die sich im sog. Plan S zusammengeschlossen haben (s. https://www. coalition-s.org/principles-and-implementation/). 


\subsection{Unzureichende Klärung rechtlicher und ethischer Aspekte}

Die unzureichende rechtliche Absicherung der Veröffentlichung und Nachnutzung von FD erweist sich häufig dann als Barriere zur Bereitstellung von FD, wenn die Forschenden am Ende eines Vorhabens zur Publikation der Daten aufgefordert werden.

Grundsätzlich bedarf es zumindest nach deutscher Rechtslage einer expliziten Erlaubnis zur Nutzung erhobener Daten für das konkrete Vorhaben ebenso wie zu einer Nachnutzung dieser Daten in einem möglichen anderen Forschungskontext. Ohne eine solche schriftlich vorliegende Erklärung, eine sog. „informierte Einwilligung“14 derjenigen, über die Daten erhoben werden (bzw. ihrer gesetzlichen Vertreter), dürfen Daten rein rechtlich noch nicht einmal in einem Repositorium aufgenommen werden. Bei nicht anonymen oder nicht vollständig anonymisierten bzw. anonymisierbaren Daten, die konkreten Personen als Aussage oder Verhalten zugeordnet werden könn(t)en, bestehen weitere rechtliche Anforderungen $u$. a. hinsichtlich der Persönlichkeitsrechte der Beteiligten. Und schließlich sind urheberrechtliche Fragen z. B. bezüglich der Bilder oder Grafiken relevant, die im Vorfeld einer Veröffentlichung und ggf. erfolgender Nachnutzung geklärt werden müssen. ${ }^{15}$ Sind die rechtlichen Anforderungen nicht vollständig erfüllt, unterbleibt häufig die $\mathrm{Pu}$ blikation der Daten. Das Resultat ist u. a. die fehlende Nachprüfbarkeit und Nachnutzung von Forschungsergebnissen.

In vielen Forschungszusammenhängen kommen aber auch ethische Fragen ${ }^{16}$ zum Tragen, z. B. die Integrität der Forschenden oder die Würde bzw. Selbstbestimmtheit von Datengebenden, über die sich Forschende bewusst werden sollten. ${ }^{17}$

$\mathrm{Zu}$ beiden Themenfelder werden zwar bei der Erstellung eines DMP konkrete Aussagen eingefordert, faktisch fühlen sich aber die meisten Forschenden hier verunsichert. Entsprechend hoch ist - im Sinne einer Barriere - der diesbezügliche Beratungsbedarf, der allerdings bislang häufig nur unzureichend erfüllt wird.

14 Vgl. beispielhaft die Hinweise des Verbundes Forschungsdaten Bildung unter https://www.forschungsdaten-bildung.de/einwilligung?la=de sowie die Angaben bei Jensen 2012, 65-67.

15 S. a. den Beitrag von Lauber-Rönsberg, Kap. 1.4 in diesem Praxishandbuch.

16 S. den Beitrag von Rösch, Kap. 1.5 in diesem Praxishandbuch.

17 Vgl. die Leitlinie 10 in den DFG-Leitlinien zur Sicherung guter wissenschaftlicher Praxis (Deutsche Forschungsgemeinschaft 2019) sowie beispielhaft den Hinweis des Verbundes Forschungsdaten Bildung unter https://www.forschungsdaten-bildung.de/ethik?la=de. 


\section{Verantwortung und mögliche Maßnahmen seitens der Stakeholder}

Aus der subjektiven Perspektive der Forschenden scheitert das Kuratieren und die Bereitstellung von FD also vordergründig zumeist an den o. g. Ursachen. Z. T. wurzeln diese im Wissenschaftssystem und seinen über Jahre entwickelten Regeln und Regularien sowie in den ggf. nicht verfügbaren Infrastrukturen. Das Kuratieren und die Bereitstellung kann durch die Veränderung der Regeln und Regularien von FD jedoch gefördert, gefordert und belohnt werden. Durch das Zusammenspiel aller Beteiligten würde möglich, was in der Literatur schon seit Jahren diskutiert wird: Bereitgestellte Hardware- und Softwarelösungen ermöglichen selbstverständlich werdende Arbeitsprozesse. Die proaktive Beratung der Forschenden und unterstützende Begleitung der FDM-relevanten Prozesse erfolgt durch Akteurinnen und Akteure, die nah an den Forschenden agieren und mit ihren Perspektiven und Interessen vertraut sind. Peers, Fachgesellschaften und die organisatorisch wie finanziell fördernden Institutionen schaffen Anreize bzw. fordern und belohnen die Bereitstellung sowie Veröffentlichung von gut kuratierten FD und deren Nachnutzung unter nachvollziehbaren Lizenzbedingungen.

Wie Stakeholder mit welchen Maßnahmen zur gewünschten Entwicklung und damit zur Überwindung und Beseitigung von Barrieren und Hemmschwellen beitragen können und sollten, wird nachfolgend dargestellt. Betrachtet werden dabei im Einzelnen jene Stakeholder, denen hierbei bislang der größte Einfluss zugerechnet wird, nämlich:

- Politik/Forschungsförderorganisationen,

- Hochschulen/außeruniversitäre Forschungseinrichtungen, hier insbesondere

- Leitungsgremien,

- Infrastruktureinrichtungen und

- Fakultäten,

- Fachgesellschaften,

- Verlage.

\subsection{Maßnahmen der Politik und der Forschungsförderer}

Bislang ist die Bereitstellung von FD auch deshalb noch keine Selbstverständlichkeit, weil eine Melange aus Hemmschwellen und Barrieren insbesondere im Publikationsmarkt dies behindert. Ordnungspolitische Vorgaben sowie damit verbundene Empfehlungen und Maßnahmen sollen dies ändern.

Die staatlicherseits u.a. über die Forschungsförderorganisationen schon seit mehreren Jahren stimulierten alternativen Formen zur Verbreitung von Forschungs- 
ergebnissen - Stichwort Open Access - wurden naheliegenderweise nach dem Konzept Open Data auf FD ausgedehnt und bilden einen wesentlichen Aspekt der sog. Offenen Wissenschaft (Open Science).

Dabei werden mit der freien Zugänglichkeit von FD mehrere ordnungspolitische Zielsetzungen verfolgt:

- Die Motivation und das Bewusstmachen, die Nachnutzung der Daten aus Forschungsergebnissen als Teil eines iterativen, im besten Fall offenen Forschungsprozesses zu sehen;

- soweit realisierbar die Wiederholbarkeit und damit auch Nachprüfbarkeit von Forschungssettings und der dabei gewonnenen Daten zum Standard zu erheben;

- $\quad$ eine Effizienzsteigerung von nationalen Forschungsaktivitäten durch deren Beschleunigung und zunehmende internationale Vernetzung sowie

- eine Beschränkung der Gesamtkosten des Publikationssystems, dessen originäre Funktion wieder stärker in den Fokus gebracht wird.

Mit der Umsetzung dieser wissenschaftspolitischen Zielsetzungen in praktische Maßnahmen sind die Forschungsförderorganisationen wie z. B. die $\mathrm{DFG}^{18}$ oder auch Forschungsgemeinschaften wie die Leibniz- bzw. Helmholtz-Gemeinschaft betraut. Sie haben hierfür - in Analogie zu ihren Pendants in anderen Ländern sowie auf der EU-Ebene - unterschiedliche Anreiz- und Steuerungsmechanismen ${ }^{19}$ entwickelt, z.B.

- die Vorgabe, DMP zu einem obligatorischen Bestandteil von Förderanträgen zu erklären und damit FD und deren längerfristige Verfügbarkeit in den Blick zu nehmen;

- die finanzielle Unterstützung der Aufbereitung von FD im Hinblick auf eine Veröffentlichung;

- die finanzielle Unterstützung einer (Daten-)Publikation als solches;

- die Vorgabe, dass Ergebnisse einer von ihnen geförderten Forschungsaktivität inklusive der zugehörigen Daten Open Access bzw. den FAIR-Prinzipien folgend veröffentlicht werden müssen, um eine möglichst breite, im Idealfall weltweite Zugänglichkeit sicherzustellen;

- die Einbeziehung von Datenveröffentlichungen in die Bewertung von Forschungsleistungen. ${ }^{20}$

18 S. den Beitrag von Putnings, Kap. 1.3 in diesem Praxishandbuch.

19 Vgl. u.a. die - allerdings unvollständige - Übersicht unter https://www.forschungsdaten.org/ index.php/F\%C3\%B6rderorganisationen.

20 Vgl. Deutsche Forschungsgemeinschaft 2015, 2. 
Gerade diese zuletzt genannte Maßnahme zielt darauf, die etablierten GatekeeperNetzwerke aus Verlagen, bedeutenden Wissenschaftlerinnen und Wissenschaftlern als Begutachtende und Herausgebende sowie den bewertenden Gremien bei Geldund Postenvergabe an Nachwuchswissenschaftlerinnen und Nachwuchswissenschaftler zu durchbrechen. Ihr Hebel ist dabei, in höherem Maße die Datenveröffentlichung als solches sowie deren Qualität als relevant zu erachten.

Die genannten Einzelmaßnahmen seitens der Forschungsförderorganisationen korrespondieren mit der vom Rat für Informationsinfrastrukturen ${ }^{21}$ (RfII) mit seinen strukturpolitisch analysierenden und stimulierenden Papieren initiierten Idee einer Nationalen Forschungsdateninfrastruktur (NFDI). ${ }^{22}$ Mit der NFDI soll dem deutschen Wissenschaftssystem ein „bundesweites, verteiltes und wachsendes Netzwerk“23 von Diensten und Beratungsangeboten für das FDM in den Jahren 2019-2028 bereitgestellt und mit jährlich bis zu 90 Millionen Euro Fördergeldern unterstützt werden. ${ }^{24}$ Ausgangspunkt dieser großangelegten strukturpolitischen Fördermaßnahme auf der nationalen Ebene ist die Erkenntnis, dass es - korrespondierend zu ähnlichen Maßnahmen auf europäischer Ebene in Form der European Open Science Cloud (EOSC) $)^{25}$ - neben den o. g. stimulierenden Einzelmaßnahmen u. a. konzertierter Aktivitäten für institutionsübergreifende Strukturen bedarf, um insbesondere disziplinbezogene Besonderheiten des FDM aufgreifen zu können und so gezielt die etablierten strukturellen Barrieren zu überwinden. Die internationale Anschlussfähigkeit der bundesdeutschen Wissenschaft soll dabei auch durch die Herausbildung einer neuen Datenkultur gefördert werden.

Auch auf der Ebene der Bundesländer sind - zeitlich z. T. sehr versetzt - Fördermaßnahmen initiiert worden. ${ }^{26}$ Schon im Jahre 2014 verdeutlichte Baden-Württemberg im Rahmen eines entsprechenden Förderkonzeptes ${ }^{27}$ die Relevanz des Handlungsfeldes FDM. Andere Bundesländer wie z. B. Hessen im Jahr 2016, ${ }^{28}$ Nordrhein-

21 S. http://www.rfii.de/de/start/.

22 RfII 2016, RfII 2017 und RfII 2018.

23 RfII 2016, 2.

24 Vgl. hierzu die Erläuterungen unter https://www.gwk-bonn.de/themen/weitere-arbeitsgebiete/ informationsinfrastrukturen-nfdi/ sowie die dort verlinkte Bund-Länder-Vereinbarung vom 26.11.2018.

$25 \mathrm{Vgl}$. für weiterführende Informationen die offizielle Website der EU unter https://ec.europa.eu/ research/openscience/index.cfm?pg=open-science-cloud.

26 Einen Überblick gibt die Seite https://www.forschungsdaten.info/praxis-kompakt/fdm-in-denbundeslaendern/.

27 Vgl. für Details „E-Science. Wissenschaft unter neuen Rahmenbedingungen“, http://mwk.baden-wuerttemberg.de/fileadmin/redaktion/m-mwk/intern/dateien/pdf/Forschung/066_PM_Anlage_E-Science_Web.pdf.

28 Vgl. Brand, Stille und Schachtner 2018. 
Westfalen in den Jahren 2017 und $2019^{29}$ oder Brandenburg $2019^{30}$ zogen zwischenzeitlich nach und legten umfangreiche Fördermaßnahmen zum fachlichen Austausch über geeignete Maßnahmen sowie zur Strukturentwicklung auf. Hierfür erfahren die länderfinanzierten Hochschulen als jene Einrichtungen, die in den meisten Fällen Erstansprechpartner für Forschende und damit ein zentraler Bestandteil der NFDI sein sollen, zunehmende Aufmerksamkeit. ${ }^{31}$

\subsection{Maßnahmen der Hochschulen / außeruniversitären Forschungseinrichtungen}

\section{Leitungsgremien}

In den 2010er Jahren griffen die Hochschulleitungen das Thema FDM auf und verabschiedeten hierzu in der Hochschulrektorenkonferenz (HRK) ${ }^{32}$ zwei grundlegende Positionspapiere. ${ }^{33}$ „Als Kernaufgaben wurden darin unter anderem die Entwicklung einer Strategie zum FDM, die Verabschiedung einer universitätsweiten Forschungsdaten-Policy und die Weiterentwicklung von Kompetenzen benannt. “34 Ziel der so angestoßenen Maßnahmen ist es,

dass die Forscherinnen und Forscher ihrer Hochschule und des gesamten deutschen Wissenschaftssystems eine Umgebung vorfinden, die ihnen ein effizientes, unkompliziertes und rechtlich abgesichertes Management der digitalen Forschungsdaten ermöglicht und damit die Grundlage für die wissenschaftliche Arbeit schafft. ${ }^{35}$

Als eine besondere Herausforderung wird dabei gesehen, dass Hochschulen - anders als die auf ausgewählte Themenfelder fokussierten außeruniversitären Forschungseinrichtungen - thematisch breit ausgerichtet und daher mit ganz unterschiedlichen Bedarfen ${ }^{36}$ sowie Datenkulturen in den verschiedenen Fachdisziplinen konfrontiert sind.

29 Nach der „Landesinitiative NFDI der digitalen Hochschule NRW“ wurde im September 2019 „fdm.nrw - Landesinitiative für Forschungsdatenmanagement“ auf den Weg gebracht; vgl. https://www.fdm.nrw.

30 Einen Überblick zu dem von 11/2019-10/2020 laufenden Projekt „Forschungsdatenmanagement in Brandenburg: Technologien, Kompetenzen, Rahmenbedingungen“ (FDM-BB) gibt https://www. forschungsdaten.org/index.php/FDM-BB.

31 Vgl. Curdt et al. 2018, 2-3.

32 S. https://www.hrk.de/.

33 S. HRK 2014; HRK 2015.

34 Helbig et al. 2019, 21.

35 HRK 2014, 3. 
Diese politische Aufforderung wird an den Hochschulen bislang sehr unterschiedlich umgesetzt. Erst allmählich scheint in den Hochschulleitungsgremien deutlich zu werden, dass es hierzu mehr als formaler Beschlüsse und der Verabschiedung einer FD-Policy ${ }^{37}$ bedarf. Die NFDI-Initiative hat diesbezüglich bei forschungsorientierten Institutionen zusätzlichen Druck erzeugt, dessen Nebeneffekt u. a. die Bildung von hochschulinternen FDM-Gruppen aus den Infrastruktureinrichtungen der Hochschulen und Vertreterinnen und Vertreter von im FDM besonders aktiver Fachdisziplinen ist. Der erwünschte Kulturwandel wird also sowohl von außen als auch oben stimuliert.

Im Zusammenspiel mit den Fakultäten haben die Hochschulleitungen auch auf der curricularen Ebene die Möglichkeit, das Thema FDM zumindest in Masterstudiengängen und Promotionskolloquien als obligatorisch zu verankern. Damit schaffen sie die Grundlage, dass die nachwachsenden Forschenden schon frühzeitig für das Thema sensibilisiert und mit praktischem Know-how zum Thema ausgestattet sind, um es in ihren eigenen Aktivitäten dann ganz selbstverständlich umzusetzen. ${ }^{38}$

Auch in den außeruniversitären Forschungseinrichtungen, die - je nach Fachdisziplin - z. T. schon seit Jahren im FDM aktiv sind, ist der Sachstand fachdisziplinbezogen unterschiedlich. Für ihre internationalen Kooperationen und Förderanträge erweisen sich etablierte FDM-Strukturen zunehmend als Voraussetzung und entsprechend selbstverständlich sind dort FDM-bezogene Vorgaben der Leitungsgremien für Workflows und kompetenzbildende Strukturen geworden. ${ }^{39}$

\section{Infrastruktureinrichtungen}

In den Hochschulen sind insbesondere die Infrastruktureinrichtungen Bibliothek und Rechenzentrum (beide z.T. sehr divers benannt) für die Umsetzung des FDM und damit die Reduzierung von Hemmschwellen und Barrieren wichtig. Im IT-Bereich stehen die campusweite Bereitstellung von Software, z. B. zur Erstellung eines DMP oder zur mittel- bis langfristigen Speicherung bzw. Archivierung von FD, sowie

36 Vgl. hierzu z. B. die in HRK 2015, 16-20, genannten unterschiedlichen „Szenarien des Forschungsdatenmanagements".

37 Beispiel für direkte Umsetzung der HRK-Überlegungen ist die Universität Münster - vgl. MeyerDoerpinghaus und Tröger 2015. Zum aktuellen Stand beim Thema Datenpolicies vgl. die Angaben unter forschungsdaten.org: Data Policies; https://www.forschungsdaten.org/index.php/Data_Policies.

38 S. die Beiträge von Fühles-Ubach und Albers, Kap. 3.1 sowie Rothfritz et al., Kap. 3.4 in diesem Praxishandbuch.

39 Vgl. z. B. die programmatische Erklärung der Helmholtz-Gemeinschaft von 2016 „Digitale Forschungsdaten offen zugänglich machen“ und die hieraus entwickelte Helmholtz-Data Federation (HDF), s. https://www.helmholtz.de/forschung/information-data-science/helmholtz-data-federation-hdf. 
der Aufbau von Strukturen zur leichten Datenablage im Vordergrund. Seitens der Bibliotheken spielt insbesondere das prozessorientierte Know-how zur Vermittlung von Kompetenzen im Umgang mit FD, deren Beschreibung mittels Metadaten sowie ggf. operative Unterstützung bei diesen Aktivitäten eine wesentliche Rolle. In welchem Maße solche infrastrukturellen Unterstützungsmaßnahmen geleistet werden können, hängt u. a. von der jeweiligen personellen Ausstattung, dem dann tatsächlich vorhandenen FDM-generischen und disziplinbezogenen Know-how sowie der Kooperationsbereitschaft bzw. einer gelungenen Arbeitsteilung zwischen diesen Akteurinnen und Akteuren ab. Tendenziell am erfolgreichsten scheinen jene Hochschulen zu sein, in denen das Thema FDM in einem Kompetenzcluster konzentriert wurde, so dass Ansprechpartnerinnen und -partner sowie Kompetenztragende für die Forschenden klar identifizierbar sind..$^{40}$ Insbesondere einführende Informationen wie z. B. sog. Coffee Lectures oder Schulungsmaßnahmen, die generisch oder disziplinbezogen angeboten werden und im Idealfall auch von anderen Hochschulen nachgenutzt werden können, ${ }^{41}$ beschleunigen diesen Veränderungsprozess, werden aber weitgehend ins Leere laufen, wenn nicht entsprechende technische und organisatorische Infrastrukturangebote seitens der Hochschulen (allein oder im Verbund) oder Forschungseinrichtungen (vgl. z. B. den Verbund Forschungsdaten Bildung) bereitgestellt werden. ${ }^{42}$

\section{Fakultäten}

Bei der Auswahl der wissenschaftlichen Mitarbeitenden wie auch von neuen Professorinnen und Professoren haben Fakultäten und ihre Gremien eine zentrale Rolle. Sie agieren auf der Grundlage von hochschulinternen Regularien wie Berufungsoder Fakultätsordnungen, in denen die formalen Auswahlkriterien für zu Berufende formuliert sind. Auf dieser Grundlage werden dann in Berufungsverfahren konkrete Anforderungen an Bewerberinnen und Bewerber entwickelt. Inwieweit hierbei auch die Veröffentlichung von FD - ergänzend zu klassischen Textpublikationen - eine Rolle spielt, wird nicht zuletzt auf dieser Ebene entschieden. Hier kann die in einer Data Policy formulierte hochschulweite Absichtserklärung zur Einbeziehung von FD-Veröffentlichungen in der Personalauswahl konkret in die Tat umgesetzt werden, wobei für eine Übergangsphase von mehreren Jahren dies vermutlich noch optional bleiben wird. Prinzipiell jedoch kann sich hier ein Mentalitätswandel über

\footnotetext{
40 Vgl. die positiven Erfahrungen aus Erlangen-Nürnberg (Putnings und Teichert 2017) oder in Delft, die Mancilla et al. 2019 berichten.

41 S. Beitrag von Helbig, Kap. 3.3 in diesem Praxishandbuch.

42 Vgl. z. B. die Aktivitäten in Aachen (Schmitz und Politze 2018; Hausen und Windeck 2018) oder insbesondere auch bzgl. der nachnutzbaren Schulungskonzepte - Berlin-Brandenburg (Helbig et al. 2019).
} 
den Stellenwert von Daten und ihrer Publikation konkretisieren. Dieser wird vermutlich durch die Einbeziehung des Publikationstyps „Forschungsdaten“ bei der Fakultätsevaluierung und ggf. damit verbundener sog. Leistungsorientierter Mittelvergabe beschleunigt.

Gleiches gilt im Prinzip auch für die Auswahlgremien außeruniversitärer Forschungseinrichtungen, bei denen der hohe Anteil von befristet beschäftigten Forschenden sowie das häufig projektorientierte und in internationalen Kontexten angelegte Forschen eine noch schnellere Einbeziehung von Datenpublikationen in die Beurteilung wissenschaftlicher Reputation ermöglicht. Inwieweit hier und im engeren Hochschulkontext tatsächlich geänderte Praktiken zum Tragen kommen, bleibt einer gesonderten Untersuchung vorbehalten.

\subsection{Maßnahmen der Fachgesellschaften}

In diversen Fachdisziplinen haben die jeweiligen Fachgesellschaften als kollektive Vertretung der jeweiligen Fachdisziplin wie auch fachlich herausragender Kolleginnen und Kollegen einen maßgeblichen Einfluss auf die fachliche Kommunikationsund Publikationskultur ihrer Mitglieder. ${ }^{43}$ Durch Anreize sowie die erklärte Forderung an Forschende, im Kontext ihrer Publikationen auch die hierzu relevanten FD bereitzustellen, beseitigen sie u. U. tradierte Praktiken und reduzieren, bestenfalls entfernen sie sogar Hemmschwellen in der Publikationspraxis. Durch die Einbeziehung eingeforderter und bereitgestellter FD in das Begutachtungsverfahren z. B. für Beiträge zu Fachtagungen sowie die selbstverständliche Möglichkeit, FD in diesem Kontext, in einer vom Verband getragenen Fachzeitschrift oder einem kooperierenden Repositorium zu veröffentlichen, fällt zudem eine strukturelle Barriere. So erfolgt für Vortragende bzw. Publizierende wie für Rezipienten die selbstverständliche Zuordnung von FD zu Publikationen. Zudem sind weiterführende Informationsdienste, wie z. B. das offene Verlinken der einer Publikation zugrundeliegenden Daten $^{44}$ oder die - ggf. synoptische - Aggregation und Auswertung solcher Daten für Metaanalysen möglich.

Fachgesellschaften haben insofern sowohl die Möglichkeit, die Publikationskultur und damit die von informellen und subjektiven Wahrnehmungen geprägten Hemmschwellen zu reduzieren bzw. zu beseitigen, als auch - sofern sie selbst Anbieter entsprechender Publikationsstrukturen sind - entsprechende Barrieren zu beseitigen.

43 Vgl. z. B. die Untersuchung im Rahmen des Projektes Options4OA durch Strecker und Pampel 2019, auch wenn diese nur einen Teil der relevanten Fachdisziplinen abdeckt.

44 Vgl. z. B. die Überlegungen und Ergebnisse im Projekt InteractOA unter https://www.zbmed.de/ forschen/abgeschlossene-projekte/interactoa/. 


\subsection{Maßnahmen der Verlage}

Verlage tragen durchaus auch Verantwortung bei der Veränderung der Veröffentlichungspraxis von FD, da sie - je nach Bedeutung und Anzahl der von ihnen herausgegebenen Zeitschriften - die gewünschte Veränderung zu einer transparenten Bereitstellung von FD mitgestalten können. Schließlich benötigen Gutachterinnen und Gutachter eingereichter Beiträge Zugang zu jenen Daten, die die Grundlage der zu publizierenden Aussagen ausmachen. Dezidierte Open-Access-Verlage, die häufig als einzelnen Hochschulen verbundene oder dort ausgegründete Verlage entstanden sind, kommt dabei eine wichtige initiale bzw. die Entwicklung befördernde Rolle zu, in dem sie u. a. mit hochschuleigenen oder fachspezifischen Repositorien kooperieren.

Die von Verlagen vorgegebenen Richtlinien und Praktiken ${ }^{45}$ sollten im Idealfall nicht zeitschriftenspezifisch sein, sondern für alle Zeitschriften des Verlages so gestaltet sein, dass sie die Workflows, Speicherorte und den konkreten Umgang mit FD klarstellen. Dies entlastet die Herausgeberinnen und Herausgeber, jeweils zeitschriftenspezifische Regelungen $\mathrm{zu}$ formulieren, auch wenn der Umgang mit FD z. T. noch disziplinspezifisch variiert. Zudem wird so deutlich, welche Linie ein Verlag beim Thema FD verfolgt. Solche Richtlinien zur Datentransparenz können nach Mellor 2018 drei Ebenen zugeordnet werden:

Level 1, Disclosure. Articles must state whether or not data underlying reported results are available and, if so, how to access them.

Level 2, Mandate. Article must share data underlying reported results in a trusted repository. If data cannot be shared for ethical or legal constraints, authors must state this and provide as much data as can be reasonably shared.

Level 3, Verify that shared data are reproducible. Shared data must be made available to a third party to verify that they can be used to replicate findings reported in the article. ${ }^{46}$

Dabei wird sofort erkennbar, dass Ebene 1 faktisch hinter dem zurückbleibt, was im Sinne der Vorgaben der meisten Forschungsfördereinrichtungen als Forderung im Raum steht. ${ }^{47}$ Das Geschäftsmodell von Verlagen muss durch solche Vorgaben keineswegs beeinträchtigt werden, denn wo FD begleitend zu Textpublikationen bereit-

45 Vgl. z. B. die nicht mehr ganz aktuelle Übersicht „Social Science Journals that have a research data policy“ unter https://jordproject.wordpress.com/project-data/social-science-journals-thathave-a-research-data-policy/. Sturges et al. 2014 haben schon frühzeitig durch eine Untersuchung im Rahmen des JoRD-Projektes auf entsprechende Defizite hingewiesen.

46 Mellor 2018.

47 Eine Auswertung der Richtlinien zur Datentransparenz bei den vier großen Verlagen Elsevier, Springer Nature, Taylor \& Francis und Wiley durch Mellor im Jahr 2018 hat ein sehr heterogenes Bild ergeben. Insofern darf die bislang erzielte oder zu erwartende Wirkung der Datenpolicies einzelner Zeitschriften wie auch Verlage nicht überschätzt werden, wie z. B. eine Untersuchung von Houtkoop et al. 2018 für die Fachdisziplin Psychologie gezeigt hat. 
gestellt werden, bleibt dabei offen. Disziplinspezifische Daten-Repositorien kommen dafür genauso in Frage wie generische Serviceangebote, wie sie z. B. von RA$\mathrm{DAR}^{48}$ bereitgehalten werden. Die Vielfalt möglicher Publikationsangebote wird durch re3data ${ }^{49}$, dem „Registry of Research Data Repositories“ transparent.

Weitergehende Überlegungen und Richtlinien, die u.a. auch Vorgaben für die Bereitstellung von Programmcode, die Zitationsweise, die Registrierung geplanter Untersuchungen (besonders für industrienahe Forschung wünschenswert) sowie die Wiederholung von Studien einbeziehen, können weitere Formen der Datenoffenheit ermöglichen. Diese sog. „Transparency and Openness Promotion Guidelines“50 wurden 2015 vom Center for Open Science ${ }^{51}$ veröffentlicht und sind nach dessen Angaben in mehr als 1000 Zeitschriften weltweit implementiert.

Verlage, aber auch publikationsunterstützende Einheiten in Infrastruktureinrichtungen und Verbünden könnten allerdings schon heute durch die Einrichtung einer Forschungsdatenredaktion die Publikation von FD sowohl Forschende, als auch die klassischen Zeitschriftenredaktionen durch die Bereitstellung hochspezialisierten Know-hows entlasten. Das von Cremer, Klaffki und Steyer am Beispiel der Geisteswissenschaften entwickelte Konzept (eine „Forschungsdatenredaktion [...] übernimmt zentrale Aufgaben im Publikationsprozess“"52) könnte somit synergetisch Hemmschwellen und Barrieren reduzieren bzw. beseitigen.

Deutlich wird, dass gerade dezidiert dem Thema Offenheit verschriebene Verlage und Organisationen die Aufbereitung und Veröffentlichung von FD ganz wesentlich vorantreiben und damit zunehmend Anforderungen aus der Wissenschaft erfüllen könnten.

\section{Fazit}

Noch sind die Forschenden in den meisten Fachdisziplinen zumeist nicht hinreichend auf die Veröffentlichung und Nachnutzung von FD bzgl. Kompetenzen und Motivation vorbereitet. Aber auch bei den Infrastrukturen sind erhebliche Defizite insbesondere im Hochschulbereich - offenbar. Die Hemmschwellen und Barrieren in Bezug auf die Veröffentlichung von FD sind insofern vielgestaltig und prohibitiv.

48 S. https://www.radar-service.eu.

49 S. http://re3data.org/.

50 Vgl. für Details https://cos.io/top bzw. für einen Kurzüberblick https://osf.io/4kdbm.

51 Das Center for Open Science (COS) ist eine forschungsnahe Infrastrukturorganisation zur Unterstützung der Open Science-Entwicklung, die u.a. die Plattform OSF zu Begleitung von transparenten Forschungsprozessen entwickelt und bereitstellt (vgl. https://cos.io/our-products/osf). David Mellor ist dort Director of Policy Initiatives des COS (Stand Oktober 2020).

52 Cremer, Klaffki und Steyer 2019, 121. 
Im Einflussbereich der diversen Gatekeeper sind vielfältige Möglichkeiten erkennbar, die vorhandenen strukturellen Barrieren sowie weiterhin bestehenden Hemmschwellen zur Veröffentlichung und freien Nachnutzbarkeit von FD zu reduzieren oder sogar mittel- bis langfristig zu beseitigen. Hierzu bedarf es nicht nur der unabdingbaren Forderungen, Vorgaben, Richtlinien und Policies, sondern konkreter Infrastrukturen und insbesondere einer aktiven Veränderungsbereitschaft der handelnden Akteurinnen und Akteure an den jeweiligen Schaltstellen. Nur so kann der angestrebte Mentalitätswandel erreicht und die Praxis offener Wissenschaft Realität werden. Der Weg dorthin scheint allerdings z. T. noch lang und mühsam zu sein.

\section{Literatur}

Letztes Abrufdatum der Internet-Dokumente ist der 15.11.2020.

Brand, Ortrun, Wolfgang Stille und Joachim Schachtner. 2018. „HeFDI - Die landesweite Initiative zum Aufbau von Forschungsdateninfrastrukturen in Hessen."o-bib. Das offene Bibliotheksjournal 5 (2/13. Juli). doi:10.5282/o-bib/2018H2S14-27, https://www.o-bib.de/article/view/ 2018H2S14-27.

Cremer Fabian, Lisa Klaffki und Timo Steyer. 2019. „Redaktionssache Forschungsdaten.“ Bibliothek Forschung und Praxis 43 (1): 118. doi:10.1515/bfp-2019-2018.

Curdt, Constanze, Marleen Grasse, Volker Hess, Nils Kasties, Ania López, Benedikt Magrean, Anja Perry, Andres Quast, Dominik Rudolph, Simone Stork, Johanna Vompras, Nina Winter. 2018. „Zur Rolle der Hochschulen - Positionspapier der Landesinitiative NFDI und Expertengruppe FDM der Digitalen Hochschule NRW zum Aufbau einer Nationalen Forschungsdateninfrastruktur.“ Zenodo (13. April). doi:10.5281/zenodo.1217526.

Deutsche Forschungsgemeinschaft, Hg. 2015. „Leitlinien zum Umgang mit Forschungsdaten.“ 30. September. https://www.dfg.de/download/pdf/foerderung/antragstellung/forschungsdaten/ richtlinien_forschungsdaten.pdf.

Deutsche Forschungsgemeinschaft, Hg. 2019. „Leitlinien zur Sicherung guter wissenschaftlicher Praxis.“ Kodex. September. https://www.dfg.de/download/pdf/foerderung/rechtliche_rahmenbedingungen/gute_wissenschaftliche_praxis/kodex_gwp.pdf.

Deutsche Forschungsgemeinschaft, Hg. 2020. „Umgang mit Forschungsdaten. DFG-Leitlinien zum Umgang mit Forschungsdaten.“ 25. Februar. https://www.dfg.de/foerderung/antrag_gutachter_gremien/antragstellende/nachnutzung_forschungsdaten/index.html\#anker62194758.

Hausen, Daniela Adele und Jürgen Windeck. 2018. „Entwicklung eines Blended Learning Kurses zum Forschungsdatenmanagement an der RWTH Aachen University." o-bib. Das offene Bibliotheksjournal 5 (3/28. September): 17-31. doi:10.5282/o-bib/2018H3S17-31, https://www.obib.de/article/view/5337.

Helbig, Kerstin, Katarzyna Biernacka, Petra Buchholz, Dominika Dolzycka, Niklas Hartmann, Thomas Hartmann, Beate Maria Hiemenz u. a. 2019. „Lösungen und Leitfäden für das institutionelle Forschungsdatenmanagement." o-bib. Das offene Bibliotheksjournal 6 (3/7. Oktober):21-39. doi:10.5282/o-bib/2019H3S21-39, https://www.o-bib.de/article/view/5505.

Hochschulrektorenkonferenz, Hg. 2014. „Management von Forschungsdaten - eine zentrale strategische Herausforderung für Hochschulleitungen. Empfehlung der 16. HRK-Mitgliederversamm- 
lung am 13.5.2014.“13. Mai. https://www.hrk.de/fileadmin/_migrated/content_uploads/ HRK_Empfehlung_Forschungsdaten_13052014_01.pdf.

Hochschulrektorenkonferenz, Hg. 2015. „Wie Hochschulleitungen die Entwicklung des Forschungsdatenmanagements steuern können. Orientierungspfade, Handlungsoptionen, Szenarien. Empfehlung der 19. Mitgliederversammlung der HRK am 10. November 2015 in Kiel.“ 10. November. https://www.hrk.de/fileadmin/_migrated/content_uploads/Empfehlung_Forschungsdatenmanagement_final_Stand_11.11.2015.pdf.

Houtkoop, Bobby Lee, Chris Chambers, Malcolm Macleod, Dorothy V. M. Bishop, Thomas E. Nichols und Eric-Jan Wagenmakers. 2018. „Data Sharing in Psychology: A Survey on Barriers and Preconditions." Advances in Methods and Practices in Psychological Science 1 (1/1. März): 70-85. doi:10.1177/2515245917751886.

Jensen, Uwe. 2012. „Leitlinien zum Management von Forschungsdaten Sozialwissenschaftliche Umfragedaten.“ GESIS Technical Reports. Juli. https://www.gesis.org/fileadmin/upload/forschung/publikationen/gesis_reihen/gesis_methodenberichte/2012/TechnicalReport_201207.pdf.

Kaden, Ben. 2018. „Warum Forschungsdaten nicht publiziert werden.“ LIBREAS. Library Ideas 33. urn:nbn:de:kobv:11-110-18452/20046-8.

Linek, Stephanie B., Benedikt Fecher, Sascha Friesike und Marcel Hebing. 2017. „Data sharing as social dilemma: Influence of the researcher's personality.“ PLOS ONE 12 (8/17. August): e0183216. doi:10.1371/journal.pone.0183216.

Lucraft, Mithu, Grace Baynes, Katie Allin, lain Hrynaszkiewicz und Varsha Khodiyar. 2019. „Five Essential Factors for Data Sharing." 2. April. doi:10.6084/m9.figshare.7807949.v1.

Mancilla, Heather Andrews, Marta Teperek, Jasper van Dijck, Kees den Heijer, Robbert Eggermont, Esther Plomp, Yasemin Turkyilmaz-van der Velden und Shalini Kurapati. 2019. „On a Quest for Cultural Change - Surveying Research Data Management Practices at Delft University of Technology.“ LIBER Quarterly 29 (1/5. August): 1-27. doi:10.18352/lq.10287.

Mellor, David. 2018. „The Landscape of Open Data Policies.“ Center for Open Science Blog. 29. August. https://www.cos.io/blog/the-landscape-of-open-data-policies.

Meyer-Doerpinghaus, Ulrich und Beate Tröger. 2015. „Forschungsdatenmanagement als Herausforderung für Hochschulen und Hochschulbibliotheken." o-bib. Das offene Bibliotheksjournal 2 (4/18. Dezember): 65-72. doi:10.5282/o-bib/2015H4S65-72.

Neuroth, Heike, Stefan Strathmann, Achim Oßwald, Regine Scheffel, Jens Klump und Jens Ludwig, Hg. 2012. Langzeitarchivierung von Forschungsdaten. Eine Bestandsaufnahme. nestor Handbücher. Boizenburg: Werner Hülsbusch. urn:nbn:de:0008-2012031401.

Putnings, Markus und Sebastian Teichert. 2017. „Erfolgreich Forschen durch Kooperation. Verknüpfung hochschuleigener Informationsstrukturen zu einem zentralen Service für Forschende. " $o$ bib. Das offene Bibliotheksjournal 4 (4/15. Dezember): 137-144. doi:10.5282/o-bib/ 2017H4S137-144.

Rat für Informationsinfrastrukturen, Hg. 2016. „Rfll-Empfehlungen ,Leistung aus Vielfalt‘ - Mai 2016.“23. Juni. urn:nbn:de:101:1-201606229098.

Rat für Informationsinfrastrukturen, Hg. 2017. „Schritt für Schritt - oder: Was bringt wer mit? Ein Diskussionsimpuls zu Zielstellung und Voraussetzungen für den Einstieg in die Nationale Forschungsdateninfrastruktur (NFDI).“ 26. April. urn:nbn:de:101:1-201705023233.

Rat für Informationsinfrastrukturen, Hg. 2018. „In der Breite und forschungsnah: Handlungsfähige Konsortien. Dritter Diskussionsimpuls zur Ausgestaltung einer Nationalen Forschungsdateninfrastruktur (NFDI) für die Wissenschaft in Deutschland.“ 4. Dezember. urn:nbn:de:101:12018120515263879760228.

Sane, Jussi und Michael Edelstein. 2015. „Overcoming Barriers to Data Sharing in Public Health. A Global Perspective." Hg. v. Centre on Global Health Security at The Royal Institute of Interna- 
tional Affairs, Chatham House. April. https://www.chathamhouse.org/sites/default/files/ field/field_document/201504170vercomingBarriersDataSharingPublicHealthSaneEdelstein. pdf.

Schmitz, Dominik und Marius Politze. 2018. „Forschungsdaten managen - Bausteine für eine dezentrale, forschungsnahe Unterstützung." o-bib. Das offene Bibliotheksjournal 5 (3/28. September): 76-91. doi:10.5282/o-bib/2018H3S76-91.

Strecker, Dorothea und Heinz Pampel. 2019. „Fachgesellschaften und Open Access in Deutschland eine Analyse zur Herausgabe von Zeitschriften.“ Zenodo (19. September). doi:10.5281/zenodo.3406288.

Sturges, Paul, Marianne Bamkin, Jane Anders und Azhar Hussain. „Zugang zu Forschungsdaten. Die Notwendigkeit von Richtlinien für Data Sharing bei Fachzeitschriften. Das JoRD-Projekt der Universität Nottingham." b.i.t.online 17 (5): 421-430.

Wiarda, Jan-Martin. 2019. „Medizin und Machtmissbrauch.“ Spektrum der Wissenschaft. Medizin (28. November). https://www.spektrum.de/kolumne/medizin-und-machtmissbrauch/ 1688058.

Wicherts, Jelte M., Marjan Bakker und Dylan Molenaar. 2011. „Willingness to Share Research Data Is Related to the Strength of the Evidence and the Quality of Reporting of Statistical Results.“ PLOS ONE 6 (11/2. November): e26828. doi:10.1371/journal.pone.0026828.

Zuiderwijk, Anneke und Helen Spiers. 2019. „Sharing and re-using open data: A case study of motivations in astrophysics.“ International Journal of Information Management 49 (1. Dezember): 228-241. doi:10.1016/j.ijinfomgt.2019.05.024. 
